\title{
Nanoscopy in the Neurosciences
}

\author{
Francisco J. Barrantes*
}

Laboratory of Molecular Neurobiology, BIOMED UCA-CONICET, A. Moreau de Justo 1600, ACF1107 Buenos Aires, Argentina.

* francisco barrantes@uca.edu.ar

Superresolution optical microscopy (nanoscopy) is increasingly being applied in the Neurosciences [1-3]. This is because nanoscopy in its various implementations covers a wide range of possibilities in the space-time palette. Among the variants of superresolution microscopy, two alternative approaches stand out: targeted strategies, like STED (Stimulated Emission Depletion superresolution microscopy) and the stochastic methods, like PALM (Photo-Activated Localization Microscopy) and STORM (Stochastic Optical Reconstruction Microscopy). The former is a "what-you-see-is-what-you-get" type of direct method, in which the fixed specimen is scanned to yield a diffraction-unlimited image. The technique is usually implemented together with confocal scans of the same specimen, thereby providing a direct comparison of the improved resolution accomplished with the nanoscopic imaging.

In the case of the single-molecule stochastic localization microscopies, the applicability of superresolution (nanoscopy) methods in the Neurosciences is directly related to the nature of the specimens (e.g. fixed or live cells, flat monolayer cells in culture or live 3D solid embryos) compounded with the type of questions one wishes to and can formulate in each case.

Why do superresolution microscopy methods find increasing applicability in the Neurosciences? Many of the interesting and still unresolved questions in the field concern structures and mechanisms occurring beyond the diffraction limit. For instance, the very nature of the synapse, a subcellular structure where receptor proteins transduce the signals coded in chemical neurotransmitters into biological responses, calls for tools capable of nanoscopic imaging with chemical specificity. If live cells are interrogated, the additional requisite of time resolution is called for. I will summarize recent developments in probes and techniques at the leading edge of current methodology in the field.

In order to understand synaptic function, it is necessary to define the supramolecular organization, dynamics and trafficking of its constituent molecules. The second part of the talk will focus on current approaches to interrogate receptor topography in static, fixed specimens where we can count individual molecules, determine their density and modes of association into nanoclusters (Figure 1) or address their dynamics using a combination of ensemble averaging methods and single-molecule experimental techniques. We can follow the translational diffusion of neurotransmitter receptors in living cells using STORM and single-molecule tracking techniques (Figure 2).

Finally, nanoscopy studies of dysfunctional synapses (i.e. "synaptopathies") will be discussed to exemplify how alteration of synaptic components is at the root of various neurodegenerative diseases affecting the peripheral and central nervous systems. 


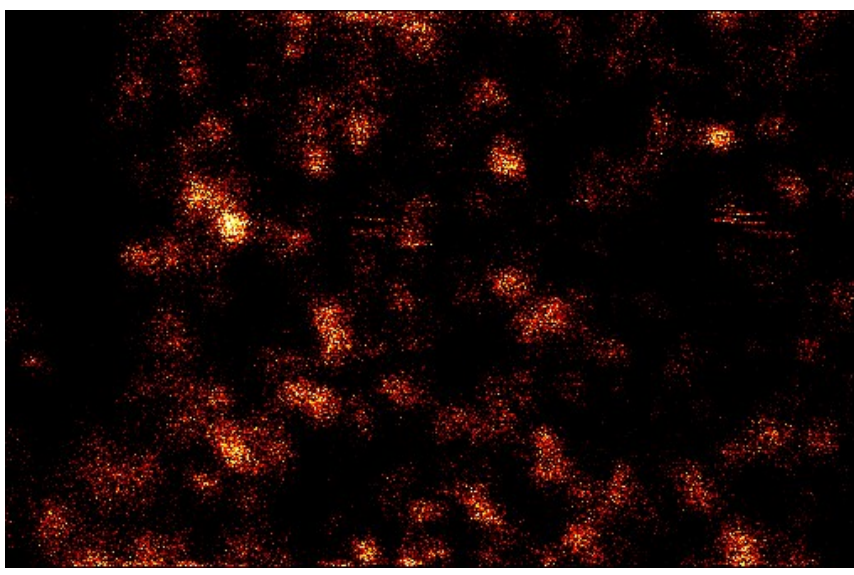

CONFOCAL

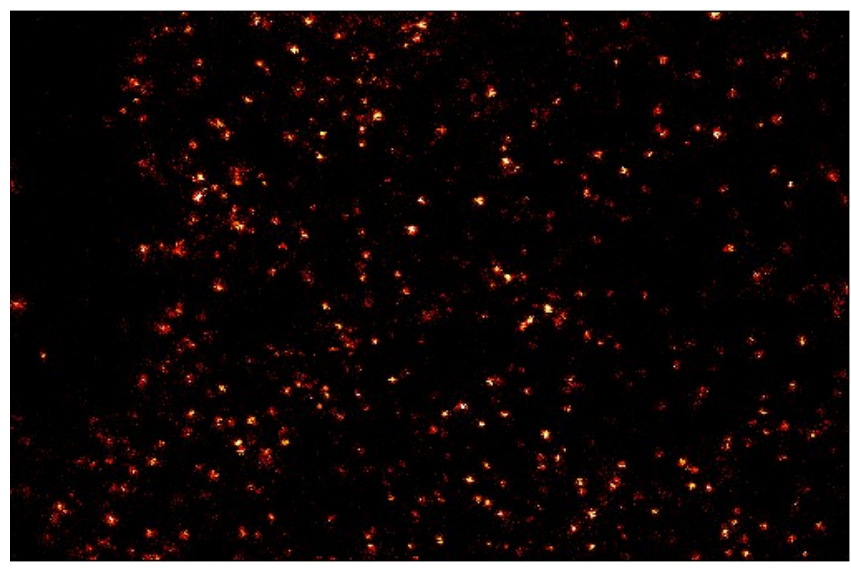

STED

Figure 1. Nicotinic acetylcholine receptor molecules at the surface of CHO-K1/A5 cells imaged with confocal and STED (Stimulated Emission Depletion superresolution microscopy), a form of targeted nanoscopy.
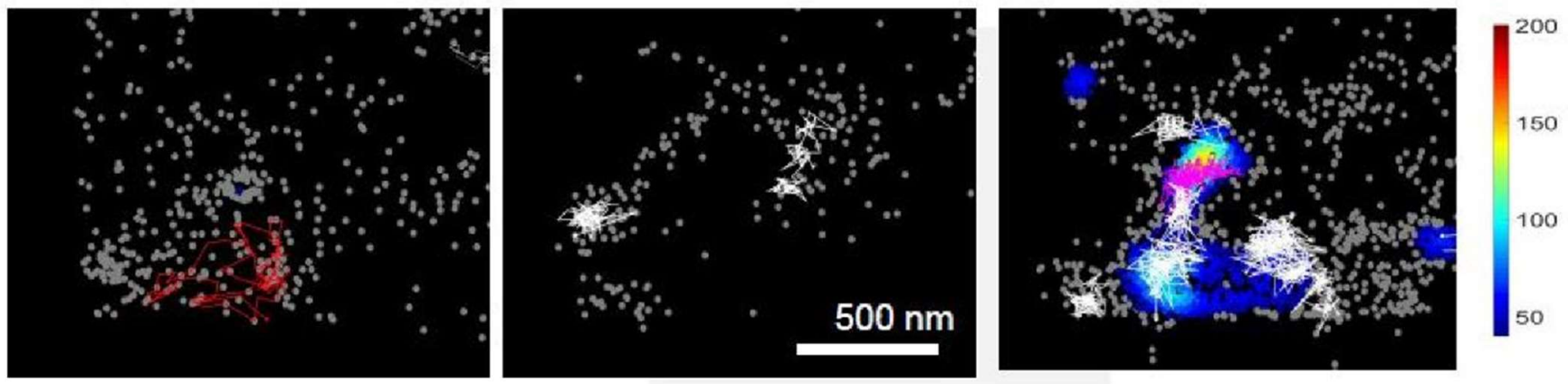

Figure 2. Nicotinic acetylcholine receptor molecules at the surface of CHO-K1/A5 cells imaged with STORM (Stochastic Optical Reconstruction Microscopy). See e.g. ref. [3] for the STORM approach and ref. [4] for the specific application to the dynamics of the nAChR. The panels show examples of individual single-molecule localizations (grey points) and traces (colored linear traces) of the singlemolecule trajectories.

\section{References}

[1] Eggeling, C., Willig, K.I. \& Barrantes, F. (2013). STED microscopy of living cells - New frontiers in Membrane and Neurobiology. J. Neurochemistry (0022.3042) 126: 203-212.

[2] Willig, K.I. \& Barrantes, F.J. (2014). Recent Applications of Superresolution Microscopy in Neurobiology. Current Opin. Chem. Biol. 20:16-21.

[3] Barrantes, F.J. (2016). Single-molecule localization superresolution microscopy of synaptic proteins. A.K. Shukla (ed.), Springer Protocols Handbooks, DOI 10.1007/8623 2016 10, Media, pp. 1-42.

[4] Mosqueira, A., Camino, P.A. \& Barrantes, F.J. (2018). Cholesterol modulates acetylcholine receptor diffusion by tuning confinement sojourns and nanocluster stability. Sci. Reports 8, 11974,

https://doi.org/10.1038/s41598-018-30384-y 